\title{
Immune targeting of the pleural space by intercostal approach
}

Georg F Weber ${ }^{1,2}$

\begin{abstract}
Background: Infectious diseases of the airways are a major health care problem world wide. New treatment strategies focus on employing the body's immune system to enhance its protective capacities during airway disease. One source for immune-competent cells is the pleural space, however, its immune-physiological function remains poorly understood. The aim of this study was to develop an experimental technique in rodents that allows for an in vivo analysis of pleural space immune cells participating in the host defense during airway disease.

Methods: I developed an easy and reliable technique that I named the "InterCostal Approach of the Pleural Space" (ICAPS) model that allows for in vivo analysis of pleural space immune cells in rodents. By injection of immune cell altering fluids into or flushing of the pleural space the immune response to airway infections can be manipulated.

Results: The results reveal that (i) the pleural space cellular environment can be altered partially or completely as well as temporarily or permanently, (ii) depletion of pleural space cells leads to increased airway inflammation during pulmonary infection, (iii) the pleural space contributes immune competent B cells during airway inflammation and (iv) inhibition of B cell function results in reduced bacterial clearance during pneumonia.

Conclusion: As the importance for in-depth knowledge of participating immune cells during health and disease evolves, the presented technique opens new possibilities to experimentally elucidate immune cell function, trafficking and contribution of pleural space cells during airway diseases.
\end{abstract}

Keywords: ICAPS, Pleural space, Immune cell trafficking, Airway inflammation

\section{Background}

Lower respiratory infections are the worlds third leading cause of death (WHO statistics 2012) and a major health care problem world wide $[1,2]$. Reasons for this are the increasing elderly population, the increase in the number of immunocompromised patients and the increase of infections with multi-resistant microbes which leads altogether to an increasing burden of the global health care system [3-8]. To counteract this process, a better understanding of the involved immune-physiological processes during pulmonary infections is needed to design more individualized approaches to the underlying disease.

One source for immune-competent cells is the pleural space - the space between the parietal and visceral

Correspondence: georg.weber@uniklinikum-dresden.de

'Department of Surgery, University Hospital Carl Gustav Carus, Technische Universität Dresden, Fetscherstrasse 74, 01307 Dresden, Germany

${ }^{2}$ Center for Systems Biology, Massachusetts General Hospital and Harvard Medical School, Boston, MA, USA pleura - each consisting of a monolayer of mesothelial cells. It contains several different leukocytes including neutrophils, monocytes and macrophages, $\mathrm{T}$ cells and $\mathrm{B}$ cells $[9,10]$. Their involvement during airway infection have been shown recently by transmigration of leukocytes into the pleural space during airway inflammation as well as regulation of neutrophilic influx by pleural space macrophages $[11,12]$. However, the involvement of pleural space immune cells during other infectious, malignant, autoimmune or allergic disease is still insufficiently understood.

For example, after performing thoracic surgery patients are regularly treated with chest tubes $[13,14]$. The purpose is to evacuate wound fluid and air from the thorax and to realign the lungs [15-17] by restoring negative pressure in the pleural space $[18,19]$. A side effect of this procedure is the constant drainage of immune competent cells from the pleural space. Recent research indicates an increased risk for pulmonary 
infections positively related to the time of chest tube treatment [20]. I therefore sought to develop an experimental technique that allows to elucidate the in vivo immunological contribution of pleural space cells during health and disease.

Two major obstacles have stood in the way of studying the immunological function of the pleural space of rodents. (i) The size: the space between the parietal and visceral pleurae is narrow, offering only a small area for manipulation. (ii) The function: during respiration, the pleural space must maintain negative pressure to align the lungs with the chest wall. In a typical approach to the pleural space investigators tend to puncture the diaphragm, which disrupts the negative pressure and causes a fatal pneumothorax. For this reason I invented a new technique that I named the "InterCostal Approach of the Pleural Space" (ICAPS) model that bypasses the diaphragm to perform in vivo analysis in rodents.

First, I mimicked the effects of chest tube treatment (which causes a drainage of immune competent cells from the pleural space) by flushing the pleural space using ICAPS and investigated the effects on the repopulation of the pleural space cellular environment. Second, I tested if the pleural space cellular environment can be altered temporarily, permanently, partially or completely by using ICAPS and third if this leads to different immune responses during airway inflammation.

\section{Methods}

\section{Animals}

C57BL/6 J (wt) were used in this study. All mice were 8-20 weeks of age at the time of sacrifice. All protocols were approved by the Animal Review Committee (AK 24-9168.11-1/2014-2) at the University Hospital Carl Gustav Carus, Technische Universität Dresden, the Government of Saxony, Germany, and the Animal Review Committee at Massachusetts General Hospital.

\section{InterCostal Approach of the Pleural Space}

To overcome the physical obstacles, and to begin exploring the immunological function and contribution of pleural leukocytes in vivo, I developed the "InterCostal Approach of the Pleural Space" (ICAPS) method. ICAPS relies on the intercostal insertion of a catheter-syringe at a low angle to the organism's thorax. The strategy bypasses the diaphragm and, because of the angle, reduces the risk of puncturing the lung. When the catheter is removed, the intercostal muscles seal the puncture canal and prevent a pneumothorax (Figure 1, Additional file 1: Video). The procedure time is 5-7 minutes. In the following, the tools and the procedure are described step-by-step.

\section{Mice and reagents}

- Male or female mice (25-30 g) or Long-Evans rats (300-325 g)

- Isoflurane (1.5 - 2.0\% (mice), 2.0 - 3.0\% (rats) mg per $\mathrm{kg}$ body weight for maintaining the anesthesia during the procedure)

- Buprenorphin (0.05 mg per kg body weight)

- Sterile alcohol prep pads

- Sterile saline solution ( $0.9 \%$ (wt/vol) saline)

\section{Equipment}

- Anesthesia platform and chamber with inhaler

- Light source

- Styrofoam pad

- Fixation tape

- Gauze pads

- Black-braided silk non-absorbable surgical spool suture 5-0 (mice)/4-0 (rats)

- Surgical instruments: dissection scissors, anatomical forceps, microsurgical scissors, and microsurgical needle holder

- Polyethylene catheter (Becton Dickinson, Intramedic, \#427401, I.D. $0.28 \mathrm{~mm}$ ) attached to a 29 gauge $0.3 \mathrm{ml}$ syringe. The tip of the catheter needs to be sharpened with an approximately $30^{\circ}$ angle by using the microsurgical scissors (Figure 1A)

- For protection of the operator and to keep the surgical field aseptic the use of gloves, face mask and surgical gown is recommended

\section{Step-by-step procedure}

a. Preparation of 3 pieces of fixation tape and installation of an accurate light source

b. Control of the anesthesia chamber and anesthesia of the rodent

c. Positioning of the rodent on the left side and fixation of the legs and the arms with the fixation tape (1) for a right antero-lateral thoracic incision

d. Cleaning of the incision area of the thorax with alcohol tips

e. Performing of a $4 \mathrm{~cm}$ long incision on the right antero-lateral thorax with the dissection scissors until reaching the thoracic wall; the attached muscles should not be damaged (2 and 3 )

f. Aspiration of the fluid or cells into the catheter syringe

g. By using the microsurgical needle holder, the sharpened tip of the catheter should be guided tangentially into the intercostal space; when the catheter is placed in the correct position it should follow the movements of the mouse thorax during breathing (4) 
A

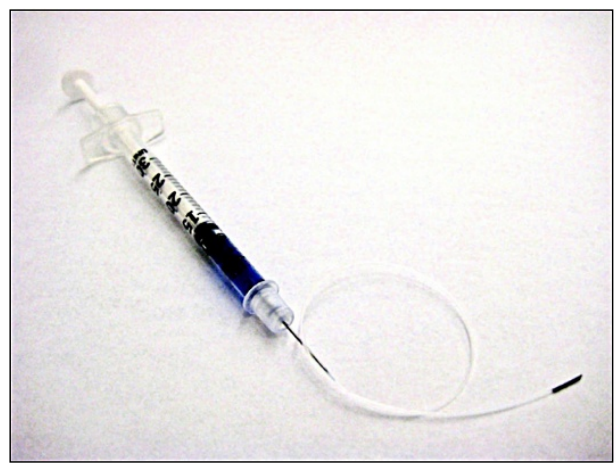

B

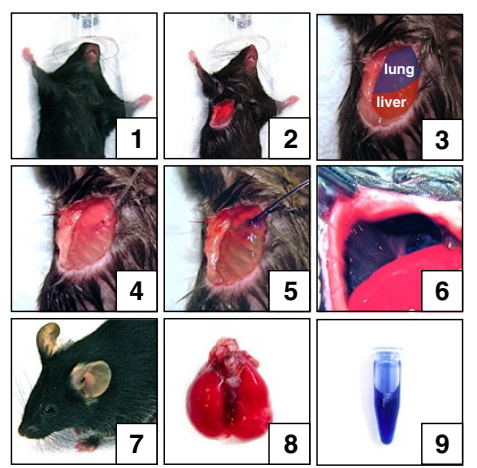

Figure 1 The "InterCostal Approach of the Pleural Space" (ICAPS) model. (A) Shown is a $0.3 \mathrm{ml}$ syringe connected to a $10 \mathrm{~cm}$ long polyethylene catheter with a sharpened tip which is the tool to approach the pleural space of rodents. (B) Illustration of the ICAPS model: (1) Mouse under isoflurane anesthesia and fixated with tape. (2) $4 \mathrm{~cm}$ long right antero-lateral thoracic incision. (3) Illustration of lung and liver seen through the thorax. (4) Insertion of the polyethylene catheter into the pleural space in a low angle to avoid damaging the lung or causing a pneumothorax. (5) Injection of the blue dye reveals the correct position of the catheter. After removal of the catheter, the skin is closed by 5/0 Ethilon suture and the mouse recovers quickly. Buprenorphin is administered i.p.. (6) To reveal the accuracy of this procedure the mouse diaphragm was explored via an abdominal incision after the procedure was performed. The blue dye can be seen through the diaphragm. (7) The mouse recovers quickly after the procedure. (8) No lung damage is observed. (9) The blue dye was recovered by flushing the pleural space revealing the correct position of the catheter.

h. Injection of fluid/cells slowly into the pleural space (5)

i. Removing the catheter tangentially in one motion

j. CAUTION: a sub-diaphragm view was performed to illustrate the successful procedure. This shouldn't be done during a planned experiment (6)

k. Performing wound closure with the a continuous suture

1. Removing of the fixation tape

m. After i.p. pain medication (buprenorphin, $0.1 \mathrm{mg} / \mathrm{kg}$ ) the rodent can be re-positioned into the cage (7)

n. CAUTION: To test for the successful procedure in this demonstration case the mouse was sacrificed. The lungs showed no damage (8) and the blue dye could be retrieved completely (9)

\section{Procedure tips and troubleshooting}

After positioning the rodent for the right antero-lateral incision, precise preparation of the thoracic wall without harming the thoracic muscles is essential. This reduces the risk for a pneumothorax after the catheter is removed as the overlaying muscles seal the puncturing canal. Undamaged muscles also help to ensure a faster recovery of the rodent. In the case of vessel injury and inadequate view on the thoracic wall, a gauze pad can be placed into the wound area. After 3 minutes the bleeding usually stops. Insertion of the catheter into the pleural space is the most important step during the procedure. The insertion should be performed in a quick, single motion. For improved control of this motion, the index finger of the left hand should stabilize the rodents right thorax while the catheter is held with the microsurgical needle holder and guided into the correct position (Figure 1B, Additional file 1: (video)). After insertion, the position of the catheter can be monitored by its movements which are aligned with the breathing excursions. If the experiment requires flushing of the pleural space the flushing solution should be injected slowly. This minimizes the risk of respiratory insufficiency. After 30 seconds the fluid should be removed entirely. This process needs to be repeated twice to efficiently deplete the cellular compartments from the pleural space. When removing the catheter, the thorax should be stabilized as well. The mouse should be monitored for 20 seconds to assure no pulmonary damage, bleeding or pneumothorax. Suture of the skin can be done with a continuous suture or clips.

\section{Complications related to ICAPS}

Analysis of intra- and post-operative complications during the procedure revealed that the pleural space can be approached in over $90 \%$ of cases without complications (Table 1). I observed three major, but thereafter mostly lethal complications:

Table 1 Analysis of complications during ICAPS

\begin{tabular}{llll}
\hline & Complication & $\mathbf{n}$ & $\%$ \\
\hline (i) & Lung injury & $4 / 110$ & 3,6 \\
(ii) & Pneumothorax & $2 / 110$ & 1,8 \\
(iii) & Bleeding & $1 / 110$ & 0,9 \\
\hline
\end{tabular}

By performing the ICAPS procedure different complications can occur. (i) In 4 of 110 cases (3.6\%) lung injury occurred. (ii) In 2 of 110 cases (1.8\%) a pneumothorax was detected. (iii) In 1 of 110 cases (0.9\%) an intra-pleural bleeding was observed. The table shows the analysis of all performed ICAPS procedures within this study. 
(i) in the unlikely event of puncturing the lung (3.6\%) during insertion of the catheter into the pleural space, the injected fluid will evacuate through mouth and nose of the animal.

(ii) after puncturing the pleural space intra-pleural bleeding $(0.9 \%)$ can occur.

(iii) after removing the catheter from the pleural space a pneumothorax (1.8\%) can occur.

In case (i) the procedure should be terminated and the mouse sacrificed. In case (ii) and (iii) proceeding with the procedure depends on the severity of the bleeding (ii) as well as the size of the pneumothorax (iii). In my experience around $50 \%$ of the mice with type (ii) and (iii) complications survived the procedure without consequences. However, after skin closure and re-placement of the mouse into the cage death of the mouse may occur in the next 24 hours. No wound infection was observed in any of the performed procedures.

\section{Intra-pleural manipulation using the ICAPS model}

Clodronate or PBS liposomes, Talcum or PBS were injected. For depletion of the pleural space cells $1 \mathrm{ml}$ PBS was injected into the pleural space and removed after 30 seconds by careful aspiration through the inserted catheter. This was performed twice. To suppress pleural B cell function $200 \mu \mathrm{g}$ of neutralizing antimouse IgM antibody (GENWAY, GWB-5A60E2) in $200 \mu \mathrm{l}$ PBS was injected intra-pleurally.

\section{Experimental design}

Regarding to the purpose of the experiment, the timing of performing ICAPS and altering the pleural space microenvironment with one of the below mentioned techniques is essential. For example, targeting the macrophage population and investigating their contribution and importance during infectious diseases (e.g. by intra-tracheal injection of Staphylococcus pneumoniae, Escherichia coli, Klebsiella pneumoniae) the injection of clodronate liposomes will deplete the entire macrophage population within 24 hours. Therefore, ICAPS would need to be performed 24 hours before induction of the airway infection. Although ICAPS is a small surgical procedure, the insertion of a catheter into the pleural space may cause alterations of the microenvironment. To control for this during experimental procedures I suggest performing ICAPS in the control group and inject sterile fluid (PBS or saline).

\section{Additional animal models and in vivo interventions}

Pneumonia models: Animals were infected intra-nasally or intra-tracheally with $20 \mu \mathrm{g}$ LPS or $5 \times 10^{6}$ CFU Escherichia coli (ATCC \# 25922) in a volume of $50 \mu \mathrm{l} \mathrm{sa-}$ line. Clinical score: The clinical score of each animal was assessed as follows (points). [a] appearance: normal (0), lack of grooming (1), piloerection (2), hunched up (3), above and eyes half closed (4); [b] behaviour - unprovoked: normal (0), minor changes (1), less mobil and isolated (2), restless or very still (3); behaviour - provoked: responsive and alert (0), unresponsive and not alert (3); [c] clinical signs: normal respiratory rate (0), slight changes (1), decreased rate with abdominal breathing (2), marked abdominal breathing and cyanosis (3); [d] hydration status: normal (0), dehydrated (5). The higher the score the worse the clinical situation of the animal. Temperature: The temperature of each animal was measured by rectal insertion of a temperature sensor while the mouse was under anesthesia.

\section{Bacteria}

Broncho-alveolar lavage (BAL) samples were diluted, plated on tryptic soy agar (BD Difco), and incubated at $37^{\circ} \mathrm{C}$. The number of bacterial colonies was assessed 12-14 h later.

\section{Murine Leukocytes}

Peripheral blood for flow cytometry was collected by aortic puncture, using a $50 \mathrm{mM}$ EDTA solution as anticoagulant. Erythrocytes were lysed using RBC Lysis Buffer (BioLegend). Total white blood cell count was obtained by preparing a 1:10 dilution of (undiluted) peripheral blood from the orbital sinus using heparin-coated capillary tubes in RBC Lysis Buffer (BioLegend). After organ harvest, single cell suspensions were obtained as follows: lungs were cut into small pieces and subjected to enzymatic digestion with $450 \mathrm{U} / \mathrm{ml}$ collagenase I, 125 $\mathrm{U} / \mathrm{ml}$ collagenase XI, $60 \mathrm{U} / \mathrm{ml}$ DNase I and $60 \mathrm{U} / \mathrm{ml}$ hyaluronidase (Sigma-Aldrich, St. Louis, MO) for $1 \mathrm{~h}$ at $37^{\circ} \mathrm{C}$ while shaking. Lungs were then homogenized through a $40 \mu \mathrm{m}$ nylon mesh. Lymph nodes were homogenized through a 40- $\mu \mathrm{m}$ nylon mesh, after which erythrocyte lysis was performed using RBC Lysis Buffer (BioLegend). The pleural space was lavaged with $2 \times 1 \mathrm{ml}$ of PBS to retrieve leukocytes. Broncho-alveolar lavage (BAL) was performed by flushing the lungs with $4 \times 1 \mathrm{ml}$ of PBS to retrieve the infiltrated and resident leukocytes. Single-cell suspensions were prepared. Total viable cell numbers were obtained using Trypan Blue (Cellgro, Mediatech, Inc, VA).

\section{Flow cytometry}

The following antibodies were used for flow cytometric analyses: anti-CD43-FITC, S7 (BD Biosciences); antiLy6C-FITC, AL-21 (BD Biosciences); anti-IgM-FITC, II/41 (BD Biosciences); anti-CD3e-FITC, 145-2C11 (BD Biosciences); anti-B220-PE, RA3-6B2 (BD Biosciences); anti-CD19-PE, 1D3 (BD Biosciences); anti-NK1.1-PE, PK136 (BD Biosciences); anti-CD49b-PE, DX5 (BD 
Biosciences); anti-90.2-PE, 53-2.1 (BD Biosciences); anti-Ly6G-PE, 1A8 (BD Biosciences); anti-Ter119-PE, TER-119 (BD Biosciences); anti-CD43-PE, S7 (BD Biosciences); anti-IgM-PerCPCy5.5, R6-60.2 (BD Biosciences); anti-MHCII-PerCPCy5.5, AF6-120.1 (BioLegend); antiCD11c-PerCPCy5.5, HL3 (BD Biosciences); anti-CD8PerCPCy5.5, 53-6.7 (BD Biosciences); anti-CD5-PECy7, 53-7.3 (eBioscience); anti-CD90.2-PECy7, 53-2.1 (BD Biosciences); anti-F4/80-PECy7, BM8 (BioLegend); antiIgM-APC, II/41 (Bd Biosciences); anti-CD43-APC, S7 (BD Biosciences); anti-Ly6C-APC, AL-21 (BD Biosciences); anti-CD25-APC, PC61 (BD Biosciences); anti-MHCIIAlexa Fluor 700, M5/114.15.2 (eBioscience); anti-CD4Alexa Fluor 700, GK1.5 (eBioscience); anti-CD19-APCCy7, 6 55 (BioLegend); anti-CD11b-APCCy7, M1/70 (BD Biosciences); anti-IgM-APCCy7, RMM-1 (BioLegend); antiCD45.2-Pacific blue (BD Biosciences); anti-CD19-Brilliant Violet 421, 6D5 (BioLegend); anti-IgM-Brilliant Violet 421, RMM-1 (BioLegend); anti-CD11b-Brilliant Violet 421, M1/70 (BioLegend). B cell populations were identified as described previously [21]. Data were acquired on a LSRII (BD Biosciences) flow cytometer and analyzed with FlowJo v8.8.6/v9.7.2 (Tree Star, Inc.).

\section{Statistics}

Results were expressed as means \pm S.D. Statistical tests included unpaired, 2-tailed Student's test using Welch's correction for unequal variances and 1-way ANOVA followed by Tukey's or Newman-Keuls Multiple Comparison Test. P values of 0.05 or less were considered to denote significance.

\section{Results}

Flushing of the pleural space and its cellular repopulation After inventing ICAPS I wondered if the pleural space cellular environment can be depleted without permanently harming the pleural space microenvironment and to mimic the postoperative situation in patients treated with chest tubes. I therefore flushed the pleural space twice with $1.0 \mathrm{ml}$ sterile PBS and was able to deplete all cellular compartments from the pleural space within 1 day. Analysis of the percentages and total amount of pleural space cells revealed that almost all pleural space macrophages were depleted within 1 day after flushing (Figure 2A). The repopulation of pleural space macrophages was gradually reaching steady state conditions 7 days after flushing. Interestingly, analysis of $\mathrm{B}$ and $\mathrm{T}$ cells revealed no dramatic percentage changes over the course of 7 days (Figure 2B). However, their amount did change dramatically on day 1 after flushing due to depletion of all cellular compartments (Figure 2C). This procedure can be performed without harming the pleural space environment and is an effective tool to temporarily deplete all cells from the pleural space.

\section{Alteration of the pleural space cellular environment}

For a better understanding of the biological contribution of immune cells during different diseases the cells of interest often need to be depleted, activated or altered during experiments. A variety of these techniques are well characterized [22-26]. Here I present three additional approaches to alter the pleural space microenvironment. The steady state environment of the pleural space contains mostly $\mathrm{B}$ and $\mathrm{T}$ cells and macrophages (Figure 2). Injection of clodronate liposomes leads to the depletion of all pleural space macrophages (Figure 3A). This procedure had no major effects on the monocyte or macrophage subsets in other compartments. Although the amount of total cells increased significantly after clodronate injection (Figure 3B) the percentage (Figure 3C) and total amount (Figure 3D) of macrophages decreased. Hence, by injection of clodronate liposomes i.pls. the pleural space macrophages can be specifically depleted. By flushing the pleural space twice with $1.0 \mathrm{ml}$ sterile PBS, all cellular compartments can be depleted without damaging the pleural spaces' biological or physiological function (Figure 2). Flushing leads to a repopulation of pleural space cells similar to the situation that patients encounter after removal of the chest tubes. By injection of Talcum, all cellular compartments can be depleted within 1 day and results in a permanent depletion of all cells in the pleural space (Figure 3).

\section{Effects of targeted versus complete cellular depletion of the pleural space}

Recent studies revealed that chest tubes increase the risk for airway infection [20]. I wondered if either depletion of pleural space macrophages or depletion of the entire pleural space cellular microenvironment can alter the course of airway infection. To evaluate this I analyzed the broncho-alveolar lavage (BAL) during steady state and 1 day after LPS i.n. challenge. During steady state conditions, neither intra-pleural injection of clodronate liposomes nor flushing of the pleural space had significant impact on the amount of immune cells in the BAL (Figure 4). However, after flushing of the pleural space, an increased influx of $\mathrm{CD}_{11 \mathrm{~b}^{+}}$cells (Figure 4A) and neutrophils (Figure 4B) into the BAL was observed 1 day after LPS i.n challenge. Interestingly, depletion of pleural space macrophages by i.pls. injection of clodronate liposomes one day prior to LPS i.n. challenge had no impact on the amount of infiltrating $\mathrm{CD}_{11 \mathrm{~b}^{+}}$cells and neutrophils as compared to controls. Analysis of the BAL revealed decreased amounts of linage cells ( $\mathrm{T}$ cells, NK cells, B cells) only after flushing of the pleural space, but no effect was observed after i.pls. injection of clodronate liposomes (Figure 4C). I therefore hypothesized that the pleural space may function as a hub for immune competent 
A

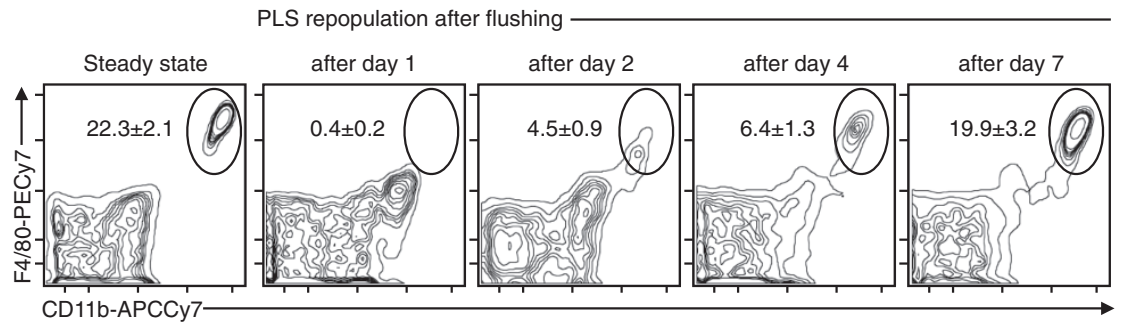

B

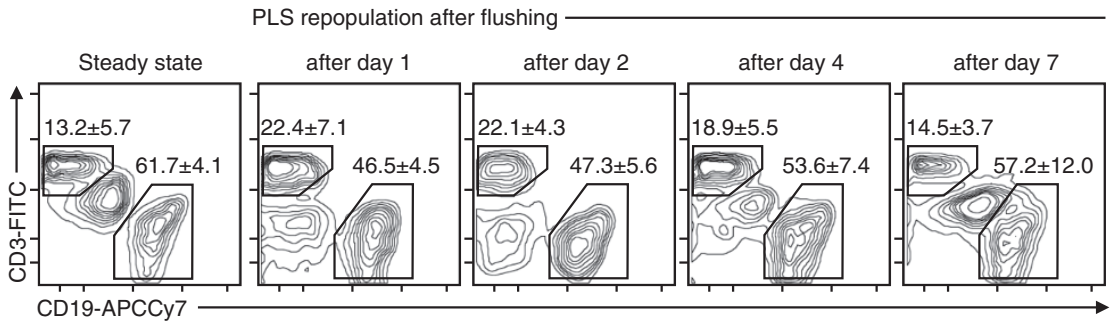

C
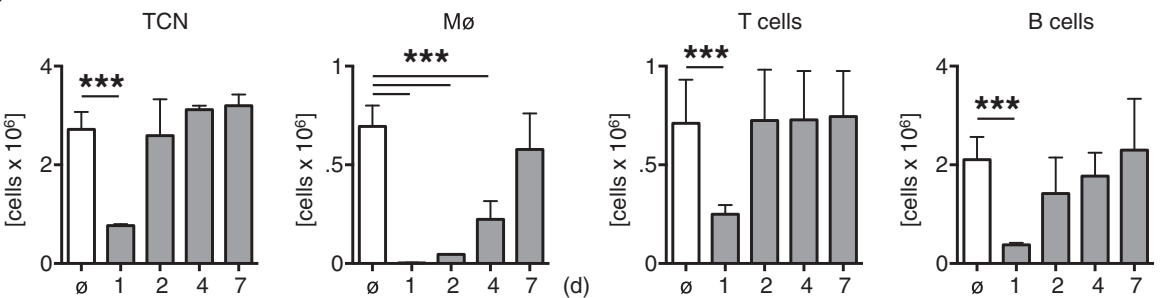

Figure 2 Time of repopulation of the pleural space cells after flushing by using ICAPS. (A) Percentages of pleural space macrophages in steady state and 1,2,4 and 7 days after flushing of the pleural space with PBS reveals an at least 7 day repopulation time. A representative analysis is shown. (B) Percentages of $\mathrm{CD}^{+} \mathrm{T}$ cells and $\mathrm{CD} 19^{+} \mathrm{B}$ cells are shown. A representative analysis is shown. (C) Analysis of the total cell numbers in steady state and 1,2, 4, and 7 days after flushing of the pleural space reveals that pleural space macrophages need 7 days to repopulate the pleural space. T and B cells repopulate within 2 days after flushing. $\left(n=6\right.$; data shown in means \pm S.D.; $\left.{ }^{* * *} p<0.001\right)$.

lymphoid cells that infiltrate the airways during an acute immune response during airway inflammation.

\section{Pleural space B1a B cells redistribute into the lungs during airway inflammation}

Based on the presented results, I tested for the immunological contribution of pleural space cells during airway inflammation. During steady state, the pleural space consists of several different cell types including monocytes and neutrophils, macrophages, T cells and B cells. Thereby, B1a B cells account for the largest cell subset (Figure 5A). I therefore sought to enumerate the amount of pleural space B1a B cells during a three day LPS i.n. challenge. Pleural space B1a B cells decreased significantly each day whereas the amount of B1a B cells increased in the lung parenchyma. No changes were found in the blood that consisted only of very few B1a B cells (Figure 5B). So far, the presented results indicate that pleural space linage cells ( $\mathrm{T}$ cells, NK cells, B cells) and especially B1a B cells may redistribute into areas of active inflammation. To directly test for this I used ICAPS and flushed the pleural space of wt mice to deplete the entire pleural space cellular microenvironment (Figure 5C). In wt mice, B1a B cells decreased significantly in the pleural space and increased significantly in the lungs 1 day after LPS i.n. challenge. In contrast, after flushing of the pleural space leading to the entire depletion of all pleural space cells, including B1a B cells, no increase of the B1a B cells in the lungs 1 day after LPS i.n. challenge was observed suggesting that B1a B cells redistribute from the pleural space into the lungs upon airway inflammation (Figure 5C). Enumeration of B1a B cells during steady state conditions and 1 day after LPS i.n. challenge revealed a significant increase of B1a B cells in the draining thoracic lymph nodes but no changes in other compartments (Figure 5D).

\section{Suppression of pleural space B cell function leads to decreased bacterial clearance}

Patients that underwent thoracic surgery regularly require chest tubes to evacuate wound fluid and air from the thorax and to realign the lungs. Although the 
A
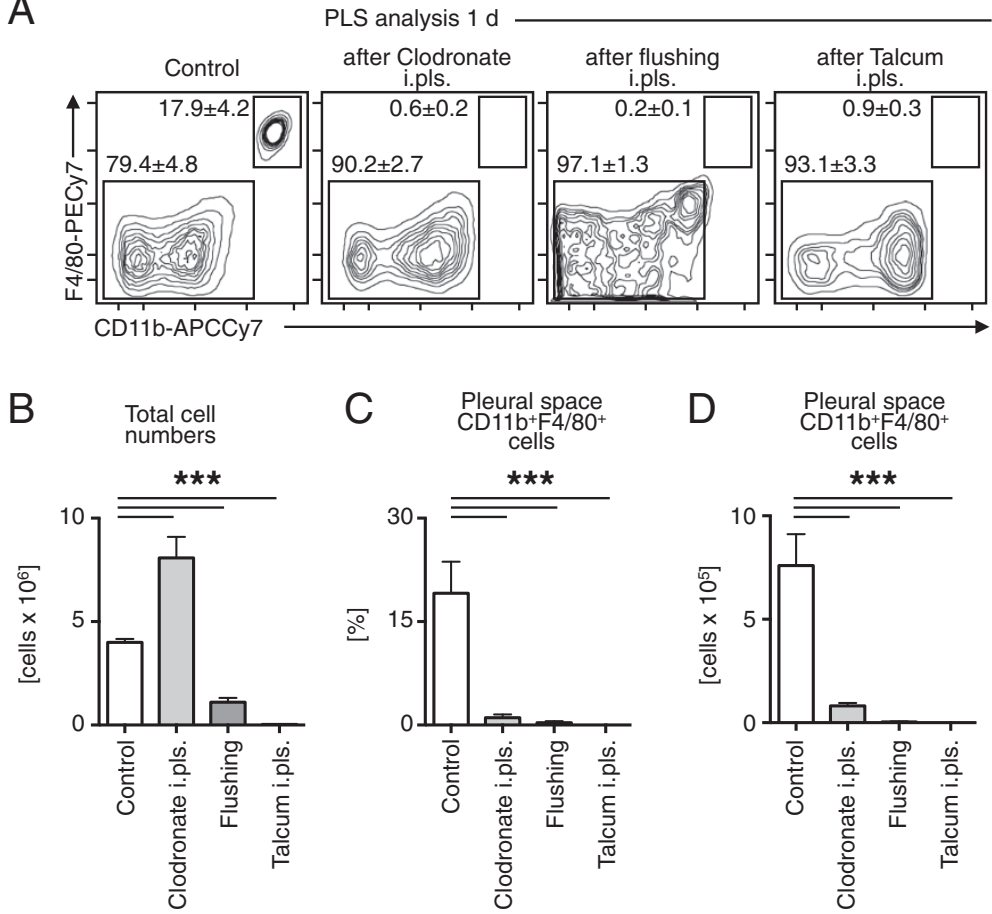

Figure 3 Altering of the pleural space cellular environment. (A) The pleural space contains CD $11 \mathrm{~b}^{+} \mathrm{F} 4 / 80^{+}$macrophages in the steady state. By using ICAPS injection of clodronate liposomes i.pls., flushing the pleural space with saline, or injection of talcum i.pls. leads to the removal of $\mathrm{CD} 11 \mathrm{~b}^{+} \mathrm{F} 4 / 80^{+}$macrophages $1 \mathrm{~d}$ after the procedure. A representative analysis is shown. (B) Total cell numbers significantly increase after injection of clodronate liposomes, and significantly decrease after flushing the pleural space or injection of talcum into the pleural space. The percentage (C) and enumeration (D) of $C D 11 b^{+} F 4 / 80^{+}$pleural space macrophages reveal their deletion within 1 day $(n=5$; data shown in means \pm S.D.; ${ }^{* * *} p<0.001$ ).

physiological function of the lungs are restored by this procedure, the immunologic microenvironment of the pleural space may be compromised. As a consequence, the susceptibility to airway infection can increase. I have already shown that depletion of the entire pleural space leads to an increase in neutrophilic influx into the BAL during airway inflammation. Recently, we have shown that B1a B cells, the major producers of natural IgM and largest cellular subset in the pleural space, can redistribute into the lungs during airway inflammation and may therefore function as an important source for protective immunity [27]. I wondered to which extent the pleural space is responsible for an sufficient protection during bacterial airway infection. To analyze the importance of pleural space B1a B cells most accurately I injected neutralizing anti-mouse IgM antibody into the pleural space of wt mice 6 hours prior to induction of bacterial pulmonary infection by i.t. injection of $5 \times 10^{6}$ CFU E.coli to selectively
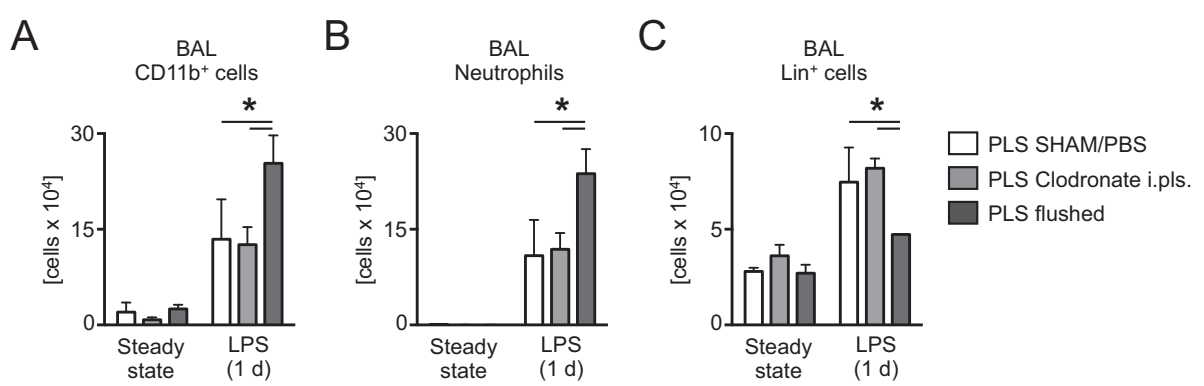

Figure 4 Depletion of the pleural space cellular environment increases the broncho-alveolar neutrophilic influx during airway inflammation. Cellular enumeration of (A) CD11 b ${ }^{+}$linage ${ }^{-}\left(\mathrm{CD} 19^{+} \mathrm{B} 22 \mathrm{O}^{+} \mathrm{CD} 90.2^{+} \mathrm{NK} 1.1^{+}\right.$Terr $\left.119^{+} \mathrm{CD} 49 \mathrm{~b}^{+}\right)$cells, (B) neutrophils (linage ${ }^{-}$Ly6G ${ }^{+} \mathrm{CD} 11 \mathrm{~b}^{+} \mathrm{MHCII}^{-} \mathrm{CD} 11 \mathrm{C}^{-} \mathrm{F} 4 / 80^{-}$Ly6 $\mathrm{C}^{\text {int. }}$ ), and (C) linage ${ }^{+} \mathrm{CD} 11 \mathrm{~b}^{-}$cells 1 day after i.pls. injection of clodronate liposomes, after flushing of the pleural space and in control mice. ( $n=4-6$; data shown in means \pm S.D.; $\left.{ }^{*} p<0.05\right)$. 
A

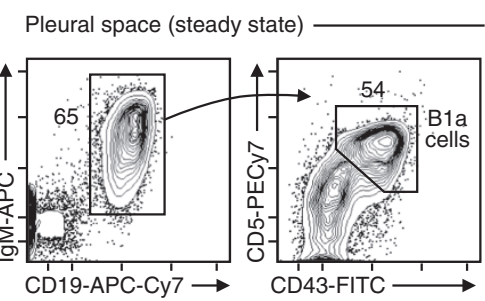

B B1a B cells after LPS i.n. $\quad$ Pleural space Lung $\quad$ Blood

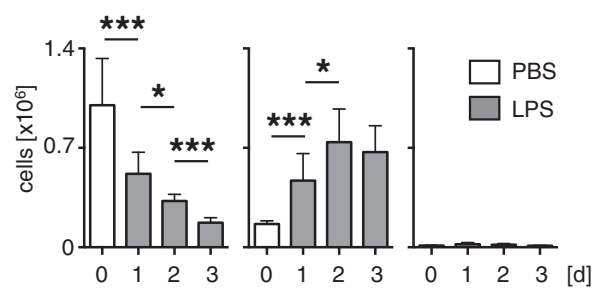

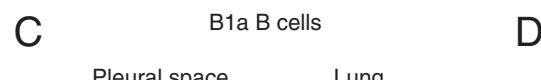

B1a B cells per organ
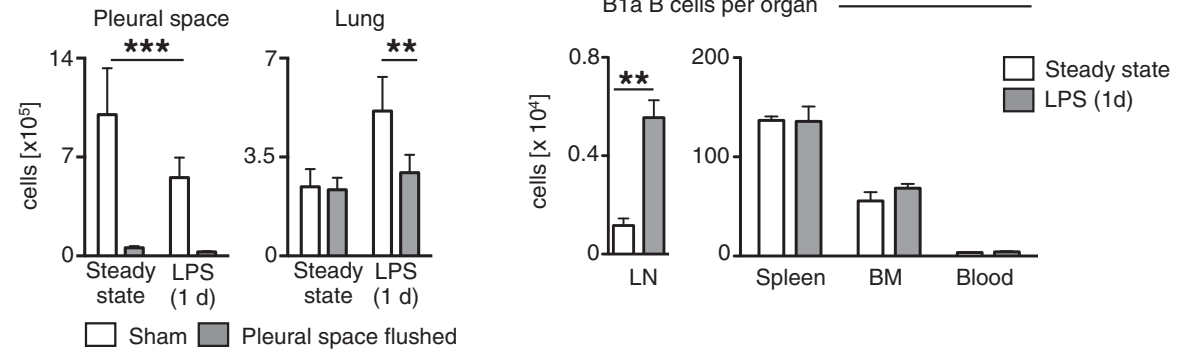

Figure 5 B1a cells evacuate the pleural space upon airway inflammation into the lungs. (A) Gating strategy of pleural space B1a B cells in the steady state. A representative analysis is shown. (B) Enumeration of B1a B cells in steady state, and 1, 2, and 3 days after LPS (20 $\mu$ g/50 $\mu$ I) i.n. challenge in the pleural space, the lungs and the blood in wt mice. (C) Enumeration of B1a B cells in the pleural space and lungs $1 \mathrm{~d}$ after LPS $(20 \mu \mathrm{g} / 50 \mu \mathrm{l})$ i.n. challenge with or without flushing of the pleural space. (D) Enumeration of B1a B cells during steady state and $1 \mathrm{~d}$ after LPS $(20 \mu \mathrm{g} / 50 \mu \mathrm{l})$ i.n. challenge in lymph nodes $(\mathrm{LN})$, spleen, bone marrow (BM), and blood. $\left(n=5-8\right.$; data are shown in means \pm S.D.; ${ }^{*} \mathrm{p}<0.05$; $\left.{ }^{* *} p<0.01 ;{ }^{* * *} p<0.001\right)$

suppress B cell function (Figure 6A) only in the pleural space. Compared to controls, i.pls. injection of neutralizing anti-mouse IgM antibody resulted in significantly increased clinical scoring and reduced body temperature indicating a more severe pulmonary infection (Figure 6B) 9 hours after induction of pulmonary infection. Bacterial counts in the broncho-alveolar lavage fluid revealed a decreased ability to clear bacterial infection after i.pls. injection of neutralizing anti-mouse IgM antibody compared to controls (Figure 6C). The results indicate that altering the immune function of pleural space B cells has impact on the hosts ability to fight airway infection.

\section{Discussion}

The results presented here identify the ICAPS model as useful technique in altering the pleural spaces' cellular environment. ICAPS allows to deplete pleural space cells partially or completely as well as temporarily or permanently. The results further reveal that depletion of pleural space cells leads to increased airway inflammation, pleural

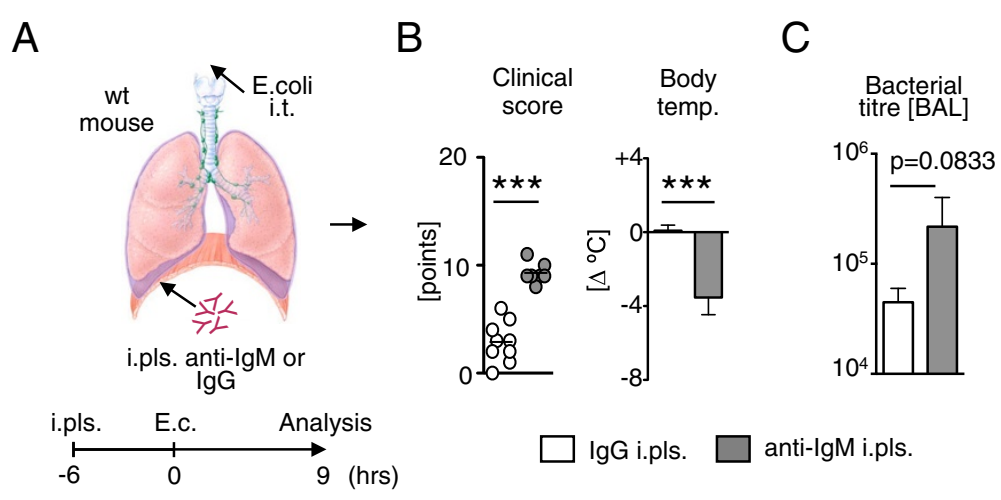

Figure 6 Suppression of pleural space B cell function by neutralizing anti-mouse IgM antibody leads to reduced broncho-alveolar bacterial clearance. (A) Intra-pleural injection of neutralizing anti-mouse $\operatorname{lgM}$ antibody or $\lg G$ isotype control into wt mice and intra-tracheal (i.t.) Escherichia coli $\left(5 \times 10^{6} \mathrm{CFU}\right)$ airway infection with analysis after 9 hrs of infection. (B) Clinical score and body temperature. (C) Bacterial titre in the BAL. $\left(n=10\right.$; data are shown in means \pm S.D.; ${ }^{*} p<0.05 ;{ }^{* *} p<0.01$; $\left.{ }^{* * *} p<0.001\right)$. 
space B cells contribute during airway inflammation and that altering their biological function leads to impaired host defense.

Our understanding of the contribution of pleural space cells during health and disease is still rudimentary. One major obstacle was the lacking possibility to experimentally investigate their immunological function. During infectious, malignant, autoimmune and allergic diseases - all of which alter the bodies immune response in different time frames - the pleural space might function as a source of immune-competent cells which influence the course of disease.

Much biology on pleural leukocytes has been inferred, but data are lacking on the cells' in vivo behavior and their contribution to airway disease. I therefore developed a new technique that I named the "InterCostal Approach of the Pleural Space" (ICAPS) to approach the pleural space of rodents in vivo. Insertion of a small catheter through the intercostal space in a low angle avoids severe complications. Bypassing the diaphragm allows thereby for a fast and easy-to-learn method to approach the pleural space (researchers as well as technicians are able to learn ICAPS within 1 day).

The experimental approach using the ICAPS model gives supporting evidence that the pleural space functions as a hub for immune cells that contribute to the immune response during airway inflammation and infection. By using ICAPS I altered the immune function of the pleural space: (i) Flushing of the pleural space led to the depletion of the entire cellular microenvironment including macrophages, $\mathrm{T}$ cells and $\mathrm{B}$ cells mimicking the postoperative chest tube treatment after thoracic surgery. (ii) Injection of neutralizing anti-mouse IgM antibody led to suppression of pleural space B cell function. Although these models do not represent the postoperative situation perfectly, both approaches resulted in an impaired control of inflammation and infection which indicates in part the cellular contribution of pleural space cells during inflammatory airway disease.

The results suggest that depletion of B1a B cells rather than macrophages lead to impaired control of airway inflammation. The reason for this could be that the pleural space functions as a source for innate like B cells [10] which participate in the early innate immune response during airway infection. Recently, we described an important role of the pleural space as a hub of IgM producing B1/IRA B cells that migrate from the pleural space into the lung during airway infection [27]. However, to which impact these cells redistribute from the pleural space into the airways remains elusive. The fact that innate like B cells are detectable in only very few amounts in the circulating system makes it likely that pleural space innate like B1a B cells contribute in an immunephysiological context to inflammatory adjacent organs and altering their function may have impact on the bodies protective immune function during inflammatory airway disease. Thus, immune targeting and activation of pleural space cells itself may increase the hosts capacity to control infection. This might have impact on the design for clinical studies investigating the function of pleural space immune cells which could lead to new therapeutic interventions for patients with pulmonary diseases.

\section{Conclusion}

Pleural space cells may be involved in several diseases. For a better understanding of their contribution, ICAPS could serve as a widely-used model and could help to answer critical questions of immune cell trafficking from the pleural space to adjacent organs (lungs, lymph nodes, heart, thymus, etc.) as well as answer questions regarding the pleural space cells' contribution during the course of pulmonary acute and chronic diseases (malignant, inflammatory, autoimmune, allergic).

\section{Additional file}

Additional file 1: Video Visualisation of the ICAPS model. The video describes the technique of the ICAPS model in rodents. Exemplary, the technique is shown on a mouse.

Competing interests

The author declares that he has no competing interests.

\section{Authors' contribution}

GFW conceived the project, designed and performed experiments, analyzed and interpreted data, constructed figures, and wrote the manuscript.

\section{Acknowledgements}

This work was supported by the German Research Foundation WE 4892/1-2 and $3-1$.

Received: 3 October 2014 Accepted: 3 February 2015

Published online: 18 February 2015

\section{References}

1. Williams BG, Gouws E, Boschi-Pinto C, Bryce J, Dye C. Estimates of world-wide distribution of child deaths from acute respiratory infections. Lancet Infect Dis. 2002:2:25-32.

2. Klevens RM, Edwards JR, Richards CLJ, Horan TC, Gaynes RP, Pollock DA, et al. Estimating health care-associated infections and deaths in U.S. hospitals, 2002. Public Health Rep. 2007;122:160-6.

3. Esperatti M, Ferrer M, Theessen A, Liapikou A, Valencia M, Saucedo LM, et al. Nosocomial pneumonia in the intensive care unit acquired by mechanically ventilated versus nonventilated patients. Am J Respir Crit Care Med. 2010;182:1533-9.

4. Mizgerd JP. Acute lower respiratory tract infection. N Engl J Med. 2008:358:716-27.

5. Venkatachalam $\mathrm{V}$, Hendley JO, Willson DF. The diagnostic dilemma of ventilator-associated pneumonia in critically ill children. Pediatr Crit Care Med. 2011;12:286-96.

6. Safdar N, Dezfulian C, Collard HR, Saint S. Clinical and economic consequences of ventilator-associated pneumonia: a systematic review. Crit Care Med. 2005;33:2184-93.

7. Vincent JL, Rello J, Marshall J, Silva E, Anzueto A, Martin CD, et al. International study of the prevalence and outcomes of infection in intensive care units. JAMA. 2009;302:2323-9. 
8. van Duijn PJ, Dautzenberg MJ, Oostdijk EA. Recent trends in antibiotic resistance in European ICUs. Curr Opin Crit Care. 2011;17:658-65.

9. Hage CA, abdul-Mohammed K, Antony VB. Pathogenesis of pleural infection. Respirology. 2004;9:12-5.

10. Baumgarth $\mathrm{N}$. The double life of a B-1 cell: self-reactivity selects for protective effector functions. Nat Rev Immunol. 2011;11:34-46.

11. Teng R, Johkura K, Ogiwara N, Zhao X, Cui L, lida I, et al. Morphological analysis of leucocyte transmigration in the pleural cavity. J Anat 2003;203:391-404.

12. Cailhier JF, Sawatzky DA, Kipari T, Houlberg K, Walbaum D, Watson S, et al. Resident pleural macrophages are key orchestrators of neutrophil recruitment in pleural inflammation. Am J Respir Crit Care Med. 2006:173:540-7.

13. Zhang Y, Li H, Hu B, Li T, Miao JB, You B, et al. A prospective randomized single-blind control study of volume threshold for chest tube removal following lobectomy. World J Surg. 2014;38:60-7.

14. Coughlin SM, Emmerton-Coughlin HM, Malthaner R. Management of chest tubes after pulmonary resection: a systematic review and meta-analysis. Can J Surg. 2012;55:264-70.

15. Cerfolio RJ, Bryant AS. The management of chest tubes after pulmonary resection. Thorac Surg Clin. 2010;20:399-405.

16. Murthy SC. Air leak and pleural space management. Thorac Surg Clin. 2006;16:261-5.

17. Utter $\mathrm{GH}$. The rate of pleural fluid drainage as a criterion for the timing of chest tube removal: theoretical and practical considerations. Ann Thorac Surg. 2013;96:2262-7.

18. Haynes D, Baumann MH. Management of pneumothorax. Semin Respir Crit Care Med. 2010;31:769-80.

19. Lai-Fook SJ. Pleural mechanics and fluid exchange. Physiol Rev. 2004:84:385-410.

20. Oldfield MM, El-Masri MM, Fox-Wasylyshyn SM. Examining the association between chest tube-related factors and the risk of developing healthcareassociated infections in the ICU of a community hospital: a retrospective case-control study. Intensive Crit Care Nurs. 2009;25:38-44.

21. Rauch PJ, Chudnovskiy A, Robbins CS, Weber GF, Etzrodt M, Hilgendorf I, et al. Innate response activator B cells protect against microbial sepsis. Science. 2012:335:597-601.

22. Leendertse M, Willems RJ, Giebelen IA, Roelofs JJ, van Rooijen N, Bonten MJ, et al. Peritoneal macrophages are important for the early containment of Enterococcus faecium peritonitis in mice. Innate Immun. 2009;15:3-12.

23. Jamur MC, Moreno AN, Mello LF, Souza Junior DA, Campos MR, Pastor MV, et al. Mast cell repopulation of the peritoneal cavity: contribution of mast cell progenitors versus bone marrow derived committed mast cell precursors. BMC Immunol. 2010;11:32

24. Schneider M. Collecting resident or thioglycollate-elicited peritoneal macrophages. Methods Mol Biol. 2013;1031:37-40.

25. Gautier EL, Ivanov S, Lesnik P, Randolph GJ. Local apoptosis mediates clearance of macrophages from resolving inflammation in mice. Blood. 2013;122:2714-22

26. Getts DR, Terry RL, Getts MT, Deffrasnes C, Muller M, van Vreden C, et al. Therapeutic inflammatory monocyte modulation using immune-modifying microparticles. Sci Transl Med. 2014;6:219ra7.

27. Weber GF, Chousterman BG, Hilgendorf I, Robbins CS, Theurl I, Gerhardt LM, et al. Pleural innate response activator B cells protect against pneumonia via a GM-CSF-lgM axis. J Exp Med. 2014;211:1243-56.

\section{Submit your next manuscript to BioMed Central and take full advantage of:}

- Convenient online submission

- Thorough peer review

- No space constraints or color figure charges

- Immediate publication on acceptance

- Inclusion in PubMed, CAS, Scopus and Google Scholar

- Research which is freely available for redistribution 